\title{
Dietary geraniol ameliorates intestinal dysbiosis and relieves symptoms in irritable bowel syndrome patients: a pilot study
}

\author{
Fernando Rizzello ${ }^{1 \dagger}$, Chiara Ricci ${ }^{2 \dagger}$, Michela Scandella ${ }^{1}$, Elena Cavazza ${ }^{1}$, Elisabetta Giovanardi ${ }^{1}$, \\ Maria Chiara Valerii ${ }^{1,3}$, Massimo Campieri ${ }^{1}$, Antonietta Comparone ${ }^{3}$, Luigia De Fazio ${ }^{3}$, Marco Candela ${ }^{4}$, \\ Silvia Turroni $i^{4}$ and Enzo Spisni ${ }^{3^{*}}$ (D)
}

\begin{abstract}
Background: (Trans)-3,7-Dimethyl-2,6-octadien-1-ol, commonly called geraniol (Ge-OH), is an acyclic monoterpene alcohol with well-known anti-inflammatory and antimicrobial properties. Ge-OH is a non-toxic compound classified as Generally Recognized As Safe (GRAS) by the US Food and Drug Administration and the European Food Security Agency. Methods: Ge-OH was orally administered at a maximum daily dose of $8 \mathrm{mg} \mathrm{kg}^{(-1)}$ body weight for four weeks in a delayed release formulation capable of reaching the colon. Fecal microbiota and blood cytokines were analyzed before and after Ge-OH treatment, as well as IBS symptomatology by using Visual Analogue Scale (VAS-IBS).

Results: The results show that orally administered $\mathrm{Ge}-\mathrm{OH}$ is a powerful modulator of the intestinal microbial ecosystem, capable of leading to increased relative abundances of Collinsella and especially Faecalibacterium, a well-known health-promoting butyrate producer consistently found to be decreased in IBS patients. Moreover, $\mathrm{Ge}-\mathrm{OH}$ strongly improved the clinical symptoms of colitis by significantly reducing the score recorded by the VAS-IBS questionnaire. Clinical improvement was associated with a significant reduction in the circulating MIP-1 $\beta$, a chemokine found to be increased in several IBS patients.
\end{abstract}

Conclusion: $\mathrm{Ge}-\mathrm{OH}$ could be a powerful component for food supplement targeted to the treatment of IBS patients.

Trial registration: ISRCTN47041881, retrospectively registered on 19th July 2018.

Keywords: Geraniol, Irritable bowel syndrome (IBS), Microbiota, Inflammation, Dysbiosis

\section{Background}

Irritable bowel syndrome (IBS) affects $9-23 \%$ of the population across the world. It is considered a debilitating disease because it strongly impairs quality of life in those affected [1] and directly impacts the working segment causing a $21 \%$ loss in productivity [2]. Abdominal pain, discomfort and bloating are common symptoms in all affected patients, but the disorder can be classified as diarrhea-predominant (IBS-D), constipation-predominant (IBS-C) and alternating stool pattern (IBS-A) based on intestinal habit [3]. IBS is

\footnotetext{
* Correspondence: enzo.spisni@unibo.it

${ }^{\dagger}$ Fernando Rizzello and Chiara Ricci contributed equally to this work.

${ }^{3}$ Department of Biological, Geological and Environmental Sciences, Biology

Unit, University of Bologna, Via Selmi 3, 40126 Bologna, Italy

Full list of author information is available at the end of the article
}

difficult to diagnose given the heterogeneity of symptoms and comorbidities that are often associated, such as gastro-esophageal reflux, non-celiac wheat sensitivity and fibromyalgia [4]. The most adopted criteria for diagnosis are the Rome III criteria, which state that a patient must have recurrent abdominal pain or discomfort at least three days/month in the last three months, associated with two or more of the following: improvement with defecation, onset associated with a change in stool frequency, onset associated with a change in form (appearance) of stools [5]. IBS therapy is mainly targeted to control patients' symptomatology and includes low-dose antidepressants, spasmolytics and 5-HT3 antagonists. However, treatments are often not effective

(c) The Author(s). 2018 Open Access This article is distributed under the terms of the Creative Commons Attribution 4.0 International License (http://creativecommons.org/licenses/by/4.0/), which permits unrestricted use, distribution, and reproduction in any medium, provided you give appropriate credit to the original author(s) and the source, provide a link to the Creative Commons license, and indicate if changes were made. The Creative Commons Public Domain Dedication waiver (http://creativecommons.org/publicdomain/zero/1.0/) applies to the data made available in this article, unless otherwise stated. 
or not tolerated by patients [6]. Different mechanisms have been implicated in IBS pathogenesis, including altered gastrointestinal motility, visceral hypersensitivity and imbalanced cytokine signaling [7] that could involve IL-1 $\beta$, IL-2, IL-4, IL-5, IL-6, IL-8, IL-10, IL-12, IL-17A, IFN- $\gamma$, TNF- $\alpha$ [7] and the chemokines MCP-1 and MIP-1 $\beta$ [8]. Even if none of them can be considered as a specific biomarker with a specific role in IBS pathogenesis, different studies indicate that a low-grade inflammation occurs in IBS patients [7, 8].

Several more recent studies have stressed the important relationship between gut microbiota dysbiosis and IBS [9]. Key findings in IBS dysbiosis include an increase in the Firmicutes to Bacteroidetes ratio, a decrease in the Lactobacilli and Bifidobacteria population, an increase in Streptococci and Ruminococcus, and a decrease of health-promoting butyrateproducing bacteria [10-12].

Probiotics and prebiotics have been investigated to evaluate their efficacy in improving symptoms in IBS patients. Systematic reviews and meta-analyses show that probiotics can significantly improve some symptoms in IBS patients [13]. However, the high variability among studies in terms of design, populations, probiotic strains and formulation used, weakens the evidence of their efficacy [14]. Prebiotics, such as inulin-type fructans and galacto-oligosaccharides, are able to modulate microbiota composition. In particular, inulin and oligofructose are known to have a bifidogenic effect. Few clinical trials have been conducted with prebiotics in IBS patients and the evidence of their efficacy is feeble [15]. In some patients, prebiotics improved the overall symptomatology but caused a worsening in bloating, and in one study prebiotic administration led to disease exacerbation, probably as a consequence of increased fermentative processes occurring in the colon [14]. Recently, the use of a wide-spectrum non-absorbable antibiotic for the treatment of IBS-associated dysbiosis has also been proposed [16].

Essential oil (EO) mixtures have been shown to play a significant role in the modulation of gut microbiota even if their mechanism(s) of action remain incompletely understood [17]. EOs have been recognized as potential new treatment options for IBS [18]. Geraniol $(\mathrm{Ge}-\mathrm{OH})$ is a naturally acyclic monoterpene component of EOs extracted from lemongrass, rose and other aromatic plants. Several studies on the biological activities of $\mathrm{Ge}-\mathrm{OH}$ have shown it to be a highly active antimicrobial compound with antioxidant and anti-inflammatory properties $[19,20]$. Ge-OH antimicrobial activities do not seem to have specific targets. Like other EOs, $\mathrm{Ge}-\mathrm{OH}$ is a hydrophobic compound able to bind to the bacterial cell wall modifying its dynamic organization, with a consequent loss of ions and ATP depletion [21,
22]. In addition to bacterial growth inhibition, $\mathrm{Ge}-\mathrm{OH}$ effectively modulates the drug resistance of several Gram-negative bacteria, such as Enterobacter aerogenes, Escherichia coli and Pseudomonas aeruginosa, by restoring drug susceptibility in strains overexpressing efflux pumps [23]. Moreover, human pathogenic bacteria are more sensitive to $\mathrm{Ge}-\mathrm{OH}$ than are commensal species even if the nature of this selectivity remains unsettled [20]. Orally administered Ge-OH (30 and $120 \mathrm{mg} \mathrm{kg}^{(-1)}$ die) strongly improved the clinical signs of colitis and significantly reduced microbial dysbiosis and cyclooxygenase- 2 (COX-2) expression in the gut wall of mice [24]. These results are in agreement with those obtained by Medicherla and co-authors [25] who found significantly reduced inflammation in the colon specimens of colitic mice after oral administration of $\mathrm{Ge}-\mathrm{OH}(50$ and $100 \mathrm{mg}$ $\left.\mathrm{kg}^{(-1)} \mathrm{die}\right)$.

Since $\mathrm{Ge}-\mathrm{OH}$ is a non-toxic compound, classified as Generally Recognized As Safe (GRAS) by the US Food and Drug Administration, and the European Food Security Agency hazard assessment conclusion for Ge-OH established a Derived No Effect Level (DNEL) of $13.5 \mathrm{mg} \mathrm{kg}^{(-1)}$ die for humans, we conducted a pilot study on IBS patients to verify the hypothesis that the anti-inflammatory and anti-dysbiotic properties of $\mathrm{Ge}-\mathrm{OH}\left(8 \mathrm{mg} \mathrm{kg}^{(-1)}\right.$ die) could improve the quality of life of these patients.

\section{Methods}

\section{Study population}

Inclusion criteria were: subjects aged 18 to 65 years, IBS diagnosis based on Rome III Criteria and BMI $\left(\mathrm{kg} \mathrm{m}^{(-2)}\right)$ $<27$ with a weight between $48 \mathrm{~kg}$ and $104 \mathrm{~kg}$. Exclusion criteria were: intolerance to lactose or known food allergies, concomitant treatment with non-steroidal anti-inflammatory drugs and antibiotics, and consumption of functional food, food supplements, probiotics and prebiotics within two months prior to the screening visit. Women in pregnancy and lactation, subjects with a diagnosis of inflammatory bowel disease or celiac disease were also excluded, together with subjects with food allergy to $\mathrm{Ge}-\mathrm{OH}$ and/or soya, subjects with serious concomitant diseases that, in the opinion of the investigator, contraindicate the patient's participation in the study and also subjects in experimental drug treatment within two months prior to the screening visit. Any other inflammatory condition was excluded in these patients by $\mathrm{C}$ Reactive Protein (CRP) and Cell Blood Count $(\mathrm{CBC})$, routinely performed as per clinical practice.

Consumption of functional food and/or food supplements (including probiotics and prebiotics) was not forbidden during the trial but it was considered a drop-out criterion. Patients were asked to maintain their normal diet during the trial. They were informed of the full 
nature and purpose of the study, and provided written informed consent before entering the trial. The study was conducted in conformity with the principles of Declaration of Helsinki and Good Clinical Practice. The sites involved in enrollment and data collection were the Inflammatory Bowel Disease Unit at S. Orsola-Malpighi University Hospital, Bologna, Italy and the Gastroenterology Unit at Spedali Civili di Brescia Hospital, Brescia, Italy. Biological samples were analyzed at Dept. of Biological, Geological and Environmental Sciences, University of Bologna.

The study was approved by the local Hospital Ethics Committees (Ethics Committee of the AOU Policlinico S. Orsola-Malpighi; CE code 100/2013/U/Sper. and Ethics Committee of the ASST Spedali Civili di Brescia; CE code NP2047).

\section{Dose selection and geraniol encapsulation}

$\mathrm{Ge}-\mathrm{OH}$ is a monoterpenoid insoluble in water with a DNEL of $13.5 \mathrm{mg} \mathrm{kg}^{(-1)}$ die for humans (General Population - Hazard via oral route, corresponding to 100 $120 \mathrm{mg} \mathrm{kg}^{(-1)}$ die in mice). Our preclinical study demonstrated $\mathrm{Ge}-\mathrm{OH}$ efficacy starting from $30 \mathrm{mg} \mathrm{kg}^{(-1)}$ die with a maximum efficacy at $120 \mathrm{mg} \mathrm{kg}^{(-1)}$ die [23], corresponding to $3-12 \mathrm{mg} \mathrm{kg}^{(-1)}$ die in humans (allometric conversion). We therefore tested a maximum dose of $8 \mathrm{mg} \mathrm{kg}^{(-1)}$ die, leaving a margin of safety with respect to the DNEL dose. After ingestion, $\mathrm{Ge}-\mathrm{OH}$ is rapidly absorbed in the intestine and quickly reaches the blood circulation [26]. In mice, $\mathrm{Ge}-\mathrm{OH}$ has maximum anti-colitis activity when delivered directly to the colon [24]. For these reasons, we proceeded with the microencapsulation of Ge-OH into soy lecithin micelle suspensions (Patent $\mathrm{n}^{\circ}$ WO 201 1/128597 Al) before filling Licaps $^{\ominus}$ capsules (Capsugel, Ploermel France) containing $150 \mathrm{mg}$ of microencapsulated $\mathrm{Ge}-\mathrm{OH}$ each. This formulation led to $\mathrm{Ge}-\mathrm{OH}$ retention in the gut, with an absolute bioavailability reduced to $50 \%$ [26].

\section{Trial design}

The study was an interventional prospective multicentric explorative non-controlled open label trial. The trial has been retrospectively registered (registration $n^{\circ}$ : ISRCTN47041881). All subjects who met the eligible criteria received a four-week treatment with $\mathrm{Ge}-\mathrm{OH}$ administered in 150-mg capsules following the dosage reported in Table 1. The treatment was followed by a four-week follow-up (Fig. 1). Study visits were scheduled at screening, at the start of treatment (V1 at T1), after four weeks of treatment (V2 at T2) and after four weeks of follow-up (V3 at T3). Clinical evaluation, physical examination, vital signs, and concomitant medications were recorded at each visit. Blood and fecal samples were collected at all visits, together with the Visual
Table $1 \mathrm{Ge}-\mathrm{OH}$ administration was twice daily after meals according to the scheme below, based on the subject's weight for a maximum dose of $10 \mathrm{mg} \mathrm{Kg}^{(-1)}$ die

\begin{tabular}{ll}
\hline Weight & Dose \\
\hline $48-59 \mathrm{Kg}$ & $3 \mathrm{cp}$ \\
$60-74 \mathrm{Kg}$ & $4 \mathrm{cp}$ \\
$75-89 \mathrm{Kg}$ & $5 \mathrm{cp}$ \\
$90-104$ & $6 \mathrm{cp}$ \\
\hline
\end{tabular}

Analogue Scale for Irritable Bowel Syndrome (VAS-IBS) questionnaire completed by subjects as described below.

\section{VAS-IBS questionnaire and IBS scoring}

At T1, T2 and T3, patients were asked to fill in the VAS-IBS questionnaire as support for clinical evaluation. The questionnaire is divided into two sections. The first consists of four questions evaluating clinical symptoms such as pain, abdominal distension, and general well-being. The total score of questions provides a value that may give an indication of disease trend as reported in Table 2. The second part of the questionnaire is a qualitative evaluation to investigate stool frequency and consistency and other disease-related symptoms. Fecal consistency was established through the Bristol stool scale.

\section{Blood collection and cytokine analysis}

Blood was collected from the antecubital fossa (left arm) by venipuncture in a Vacutainer ${ }^{\circ}$ (BD Science) containing ethylenediaminetetraacetic acid at $\mathrm{T} 1, \mathrm{~T} 2$ and $\mathrm{T} 3$. Blood samples $(5 \mathrm{ml})$ were kept at $4{ }^{\circ} \mathrm{C}$ for $1 \mathrm{~h}$, and then centrifuged at $1000 \mathrm{~g}$ for $15 \mathrm{~min}$. Plasma was collected and stored at $-80^{\circ} \mathrm{C}$ until cytokine and chemokine analyses.

Plasma cytokines were quantified in triplicate (plasma dilution 1:4) using a customized detection 13-plex panel (IL-1 $\beta$, IL-2, IL-4, IL-5, IL-6, IL-8, IL-10, IL-12, IL-17A, IFN- $\gamma$, MCP-1, MIP-1 $\beta$, TNF- $\alpha$ ) purchased from BioRad (USA). The assays were performed in 96-well filter plates by multiplexed Luminex ${ }^{\circ}$-based immunoassay following the manufacturer's instructions. IL-1 $\beta$, IL-5, IL-8, IL-10, IL-17A, IFN- $\gamma$ and TNF- $\alpha$ were read in the Luminex ${ }^{\circ}$ using the high sensitivity mode. Microsphere magnetic beads coated with monoclonal antibodies against the

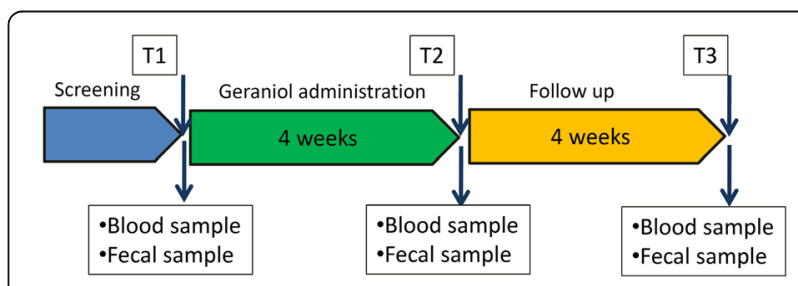

Fig. 1 Flowchart of study protocol 
Table 2 VAS-IBS total score and relative trend of IBS disease

\begin{tabular}{ll}
\hline VAS-IBS Score & IBS disease \\
\hline$<75$ & Remission \\
$75-175$ & Mild \\
$175-300$ & Moderate \\
$>300$ & Severe \\
\hline
\end{tabular}

different target analytes were added to the wells. After 30-min incubation, the wells were washed and biotinylated secondary antibodies were added. After further incubation for $30 \mathrm{~min}$, beads were washed and then incubated for $10 \mathrm{~min}$ with streptavidin conjugated to the fluorescent protein phycoerythrin (streptavidin/phycoerythrin). After washing, the beads (a minimum of 100 per analyte) were analyzed in a BioPlex 200 instrument (BioRad). Sample concentrations were estimated from the standard curve using a fifth-order polynomial equation and expressed as $\mathrm{pg} / \mathrm{ml}$ after adjusting for the dilution factor (Bio-Plex Manager software 5.0). Samples below the detection limit of the assay were recorded as zero, while samples above the upper limit of quantification of the standard curves were assigned the highest value of the curve. The intra-assay coefficients of variability averaged $12 \%$.

\section{Fecal microbiota analysis}

Fecal samples were collected from IBS patients at T1, T2 and $\mathrm{T} 3$ and stored at $-20^{\circ} \mathrm{C}$ until DNA extraction. Nucleic acids were extracted from $250 \mathrm{mg}$ of sample using PowerSoil ${ }^{\circ}$ DNA Isolation Kit (MoBio Laboratories, Inc., CA, USA) according to the manufacturer's recommendations. DNA sample quality was checked using a Nanodrop $100^{\text {ru }}$ (NanoDrop Technologies, Wilmington, DE, USA). The hypervariable region of the 16S rRNA gene was amplified using the universal forward primer $16 \mathrm{~S} 27 \mathrm{~F}$ and reverse primer $\mathrm{r} 1492$, and then sequenced on a 454 GS FLX Titanium and FLX+ (Roche, Basel, Switzerland) sequencing system, at MR DNA (Molecular Research LP, Shallowater, TX, USA). Sequencing reads were deposited in SOURCEFORGE (https://sourceforge.net/projects/geraniol-in-ibs/).

Publicly available $16 \mathrm{~S}$ rRNA gene sequence data from 12 Italian Caucasian subjects (mean age, 33 years; 7 females and 5 males) were retrieved (NCBI Sequence Read Archive, BioProject ID PRJNA340060) and used as a control to characterize microbial dysbiosis in IBS [27]. These subjects were healthy at the time of fecal sample collection, and they had no history of major gastrointestinal disorders All these subjects had not received antibiotics, probiotics or prebiotics for at least three months before sampling.

Fecal specimens from healthy controls were collected and processed in the same way. All sequence data were processed by using comparable bioinformatics pipelines [28]. Briefly, quality-filtered reads were clustered into OTUs at 97\% similarity threshold using UCLUST [29]. Singleton OTUs and chimeras were removed. Taxonomy assignment was performed using the RDP classifier and BLASTn against the Greengenes database. Alpha diversity was computed using the Simpson index. Beta diversity was estimated by calculating Euclidean distances between genus-level microbial profiles.

\section{Safety assessments}

Treatment-emergent adverse events were monitored throughout the study. The relation between adverse events and the study compound was classified by the investigators as (i) definitely related, (ii) probably related, (iii) possibly related, (iv) unknown or unable to determine, (v) probably not related, and (vi) definitely not related. The first four categories were considered study drug-related adverse events. A physical examination was performed at each visit. Laboratory tests (hematology/biochemistry/urinalysis) were planned in case of adverse events.

\section{Statistical analysis}

Being a pilot study, the number of patients to be enrolled was calculated based on similar published studies [30]. Continuous variables are expressed as mean \pm SEM of at least three independent determinations. Once the homogeneity of variances (homoscedasticity, $F$ test) had been verified, statistical differences between groups were determined by Student's T test using GraphPad Prism 6 (GraphPad Software Inc., CA, USA). Differences were considered statistically significant at $P<0.05$. Categorical variables are expressed in total counts and \% of counts, and were compared using $X^{2}$ test. Differences were considered statistically significant at $P<0.05$. All microbiota statistical analyses, including principal component analysis (PCA) of Euclidean distances between genus-level profiles, permutation tests with pseudo-F ratios (to assess the significance of data separation in PCA space) and non-parametric tests (Wilcoxon test, paired or unpaired as needed, for alpha and beta diversity, and relative abundances of bacterial taxa), were performed in $\mathrm{R} 3.3 .2$ using $\mathrm{R}$ studio 1.0.136. In cytokine multiple comparisons, statistical analysis was performed by correcting $P$ values by using the Benjamini-Hochberg method. A corrected $P$ value $<0.05$ was considered statistically significant. We did not perform statistical analysis, nor evaluate Simpson index or Euclidean distances in the IBS-C subtype group due to the small sample size $(n=3)$. 


\section{Results}

\section{Patients enrolled and clinical evaluation}

The cohort of 19 IBS Italian patients (Caucasian, 8 male, 11 female, mean age, 38.84 years) included nine IBS-D, seven IBS-A and three IBS-C. None of them was post-infective IBS. None of them was following a particular diet for IBS (i.e. low carb, low gluten, low FODMAPs). No concomitant nor additional therapies, related to IBS management, were taken by patients from T1 to T3. As reported by treated patients and recorded by VAS-IBS score, individual symptoms significantly improved during the treatment period in all IBS subtypes. Improvements in bloating and intestinal regularity were declared by almost all patients. VAS-IBS score significantly decreased after treatment from a severe mean condition $(309.95 \pm 81.23)$ to a moderate mean condition $(216.47 \pm 87.26)$ (Fig. 2). This decrease was extremely statistically significant $(P<0.001)$. After four weeks of follow-up, the VASIBS score reached a higher mean value, still remaining in the moderate mean condition (261.42 \pm 95.86) but losing statistical significance compared to T1 $(P=0.507)$. Analyzing the different IBS subtypes, at T2 both IBS-D and IBS-A subtypes showed decreased scores $(258.78 \pm 84.25$ and $184.43 \pm 89.97$, respectively) that were statistically significant $(P=0.0421$ and $P=0.0472$, respectively), while only the IBS-D subtype maintained a VAS-IBS score significantly decreased at T3 $(248.66 \pm 89.92 ; P=0.030)$.

\section{Cytokines and inflammatory markers}

Among all circulating cytokines and chemokines evaluated, IL-1 $\beta$, IL-5, IL-10 and TNF- $\alpha$ showed undetectable values in most patients. At T2, only MIP-1 $\beta$ (Fig. 3) showed a statistically significant reduction $(P=0.016)$, while the MCP-1, IL-6 and IL-17A decreases at T2 were

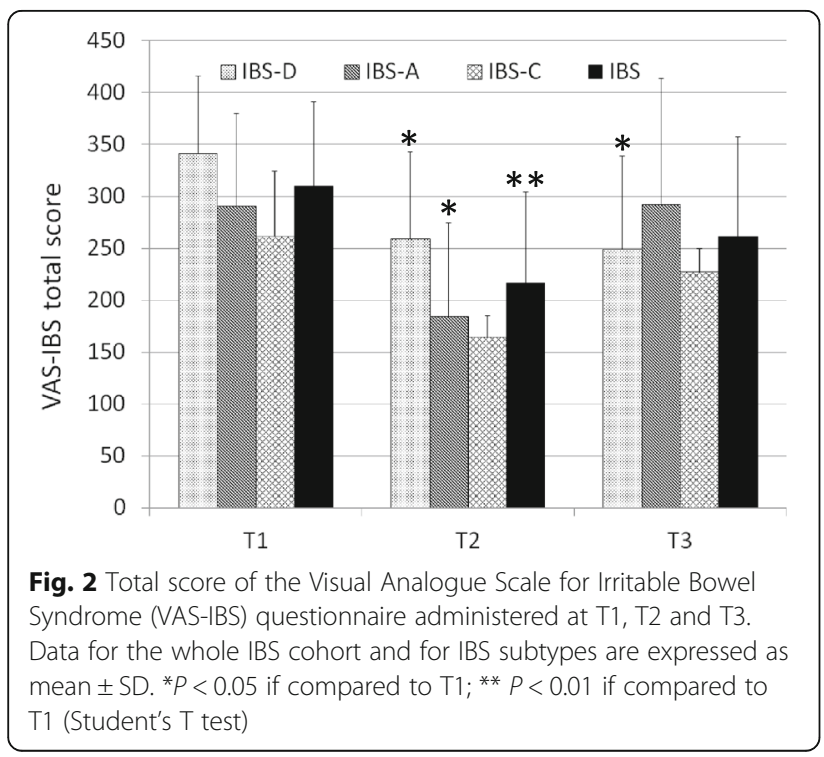

only close to achieving statistical significance $(P=0.054$, $P=0.059$ and $P=0.061$, respectively). Moreover, IL-6 plasma concentrations were detectable $(>0.5 \mathrm{pg} / \mathrm{ml})$ only in 10 patients out of 19 . At T3, all cytokine values were similar to those measured at T1, demonstrating that the overall systemic anti-inflammatory effect disappeared four weeks after treatment discontinuation. The only exception was IL-17A, which decreased with respect to T2 but without reaching statistical significance $(P=0.057)$. Statistical analysis performed on IBS subtypes did not show any other significant differences. At T2, MIP-1 $\beta$ was significantly decreased only in IBS-D subtype group $(P=0.032)$, even if this chemokine showed an evident decrease in all IBS subtypes.

\section{Gut microbiota modulation}

Consistent with the available literature repeatedly reporting reduced biodiversity of the intestinal microbiota in most human diseases [31], the Simpson index value was found to be significantly lower in our IBS cohort if compared to healthy controls (HC) $\left(P=9 \times 10^{-6}\right)$ (Fig. 4a). Moreover, based on our findings, the gut microbiota of IBS-D patients was less biodiverse than that of IBS-A patients $(P=0.010)$. Similarly, the Euclidean distance ordination showed separation between our IBS patients and $\mathrm{HC}$ (permutation test with pseudo-F ratios, $P=2 \times$ $10^{-5}$ ) (Fig. 4b), with IBS characterized by a far greater interpersonal variation in microbiota structure (mean Euclidean distance \pm SEM, IBS vs HC, $55.15 \pm 2.23$ vs $17.34 \pm 0.50$; Wilcoxon test, $\left.\mathrm{P}=9 \times 10^{-28}\right)$. IBS-A and IBS-D samples were largely overlapping, with a trend towards greater interpersonal microbiota variation in the IBS-D subtype group (Fig. 4b). Genus-level taxonomic comparisons uncovered IBS-specific microbial signatures, including a considerable enrichment in the Bacteroidetes members Prevotella and Bacteroides, and in Eubacterium and Megamonas, and a low representation of commonly considered health-associated microbiota members such as Bifidobacterium, Faecalibacterium, Blautia and Dorea, as well as Collinsella $(P<0.05)$ (Fig. 4c). In addition, the genera Escherichia (mean relative abundance in IBS, 1.08\%) and Alistipes $(2.74 \%)$ were only detected in the fecal microbial communities of our IBS cohort.

After four-week $\mathrm{GeOH}$ administration, the gut microbiota biodiversity in IBS patients tended to increase, even if the difference was not statistically significant (Simpson index at T2, mean \pm SEM, $0.69 \pm$ $0.05 ; P=0.4)$. Likewise, a slight separation between T1 and T2 samples was apparent in the PCA plot, with a shift towards decreasing PC2 values, but statistical significance was not achieved (mean PC2 coordinate \pm SEM, T1 vs T2, $0.60 \pm 0.62$ vs $-0.30 \pm$ $0.63 ; P=0.14$ ) (Fig. 5a). At the compositional level, 


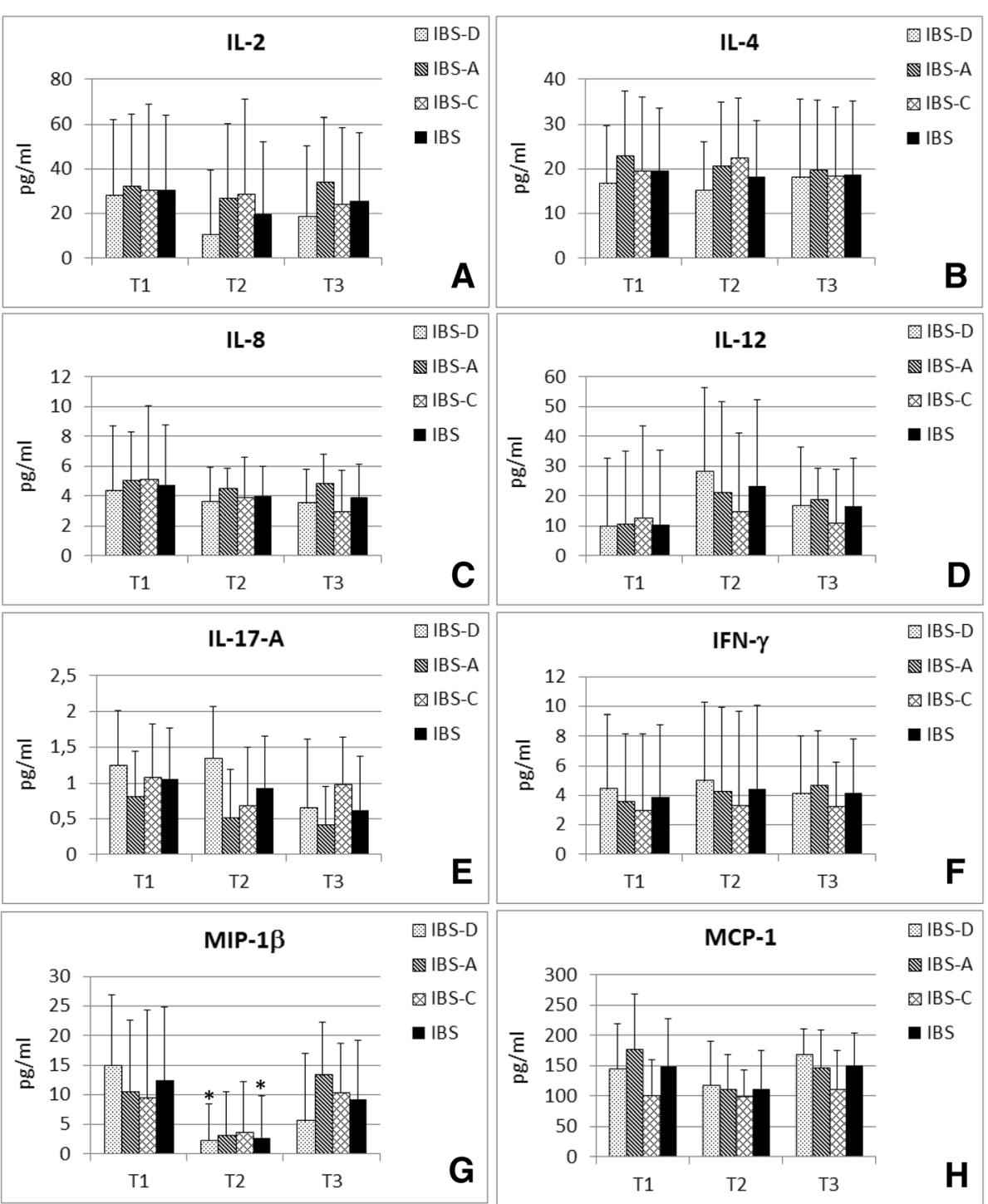

Fig. 3 Plasma cytokine variations measured at T1,T2 and T3. Cytokines were determined using a 13-plex mouse bead immunoassay kit. Levels of IL-2 (a), IL-4 (b), IL-8 (c), IL-12 (d), IL-17A (e), IFN-Y (f), MIP-1 $\beta$ (g) and MCP-1 (h) are shown for the whole IBS cohort and for IBS subtypes. Data are expressed as mean \pm SD of at least three replicates. Calculated $P$ values were corrected for multiple comparisons by using the Benjamini-Hochberg method. ${ }^{*} P<0.05$ if compared to $\mathrm{T} 1$

the gut microbiota of Ge-OH-receiving IBS patients showed a significant increase in the relative abundance of Collinsella and Faecalibacterium at T2 compared to the baseline (for both, $P=0.04$; Fig. $5 \mathrm{~b}$ ), while no differences were observed for Escherichia and Alistipes. the relative abundances of Collinsella was found significantly increased $(P=0.03)$ at T2 in IBS-A subtype, while no other significance was observed in the different IBS subtype groups. Although not significant, trends towards increased proportions of Bifidobacterium, Blautia and Faecalibacterium, and decreased percentages of Bacteroides and Prevotella were also observed $(P \leq 0.2)$ (Fig. 5b).
At follow-up after a further four weeks, the alpha diversity value was still comparable to that detected after the intervention (Simpson index at T3, mean \pm SEM, $0.70 \pm 0.05$ ). Similarly, follow-up samples generally overlapped with samples at T2 in the PCA plot, still showing lower PC2 coordinates compared to the baseline (mean \pm SEM, $-0.30 \pm 0.63$ ) (Fig. 5a). No difference was observed in the genus-level compositional structure of the gut microbiota between T2 and T3 samples. Compared to the baseline, the trends towards increased percentages of Blautia and decreased proportions of Prevotella were still maintained $(P \leq 0.3)$ (Fig. $5 b)$. 
A

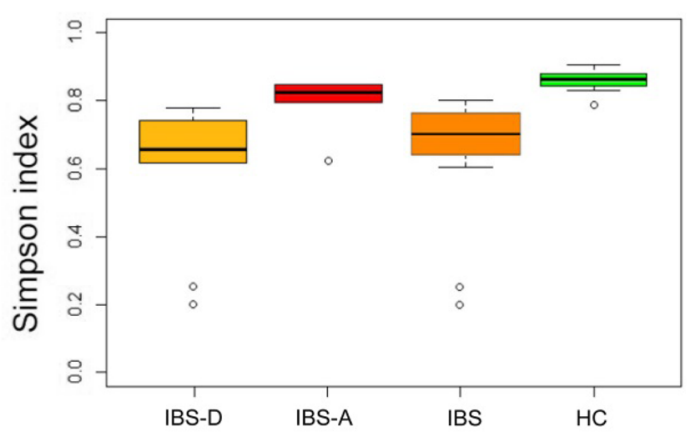

B

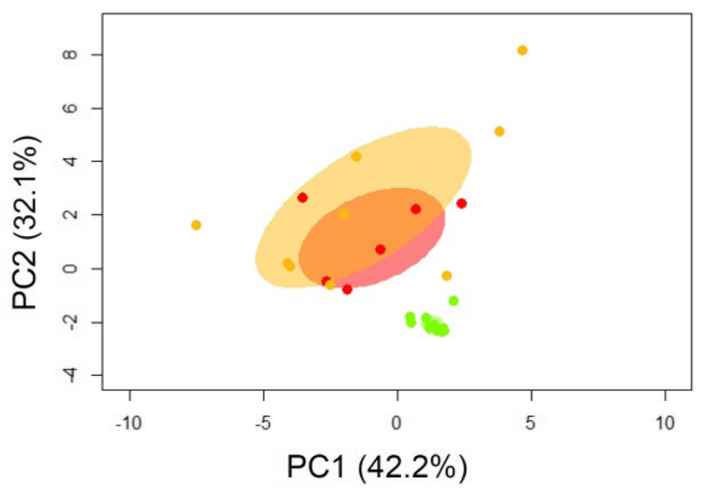

C

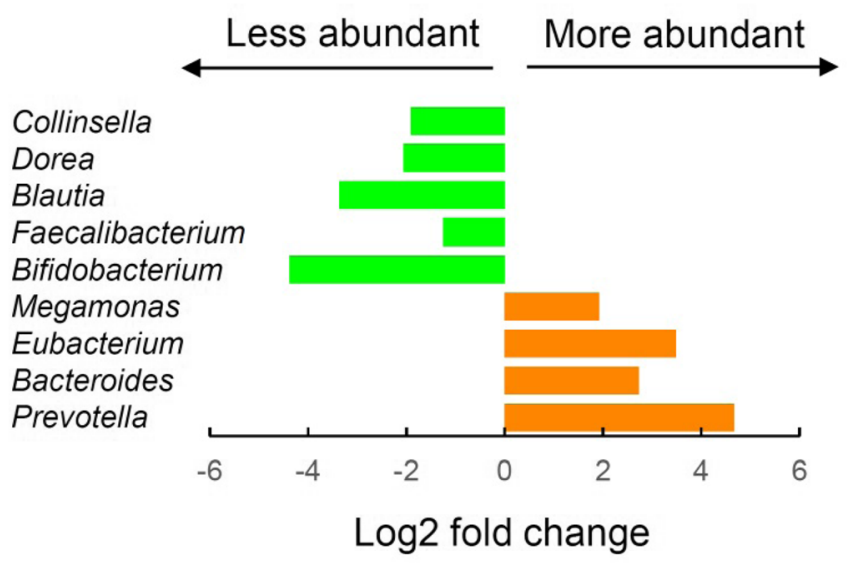

Fig. 4 Gut microbiota signatures in IBS. a Box plots showing the distribution of Simpson diversity values in IBS patients (IBS-A, red; IBS-D, orange; IBS-C, black) and healthy controls (HC, green). A significant difference between IBS patients and HC was found (Wilcoxon test, $P=9 \times 10^{-6}$ ). $\mathbf{b}$ Principal component analysis of Euclidean distances between the genus-level intestinal microbial profiles (same color code as in A). Ellipses include 99\% confidence area based on the standard error of the weighted average of sample coordinates. A significant separation between IBS and $\mathrm{HC}$ samples was found (permutation test with pseudo-F ratios, $P=2 \times 10^{-5}$ ). c Genus-level microbial signatures of IBS, shown as Log2-fold changes between IBS and control samples. Orange, taxa more abundant in IBS; green, taxa more abundant in HC. Wilcoxon test, $P<0.05$

\section{Safety and tolerability}

No adverse events related to $\mathrm{Ge}-\mathrm{OH}$ administration were reported during the study. Ge-OH orally administered using soy lecithin micelles was well-tolerated by patients.

\section{Discussion}

$\mathrm{Ge}-\mathrm{OH}$ is a natural monoterpene classified in the GRAS category [32]. Its therapeutic potential includes anti-inflammatory, antioxidant, and antibacterial effects, often 
A

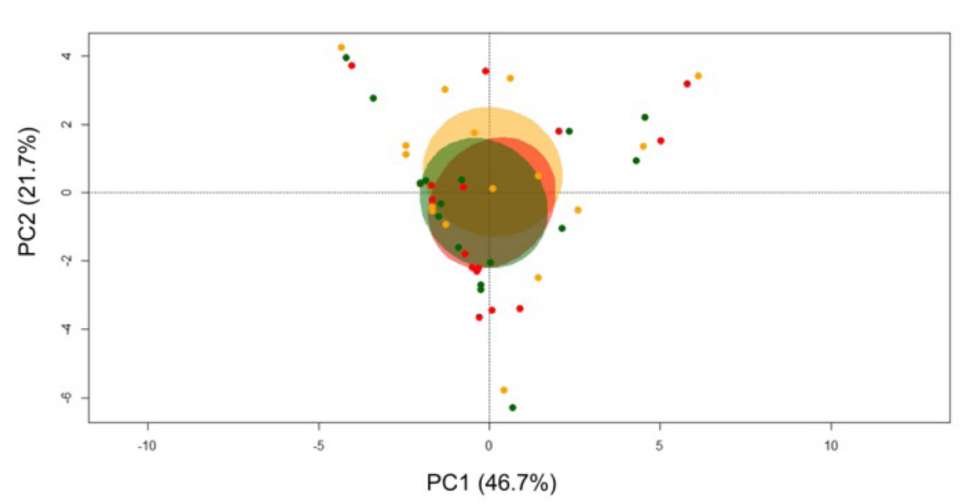

B
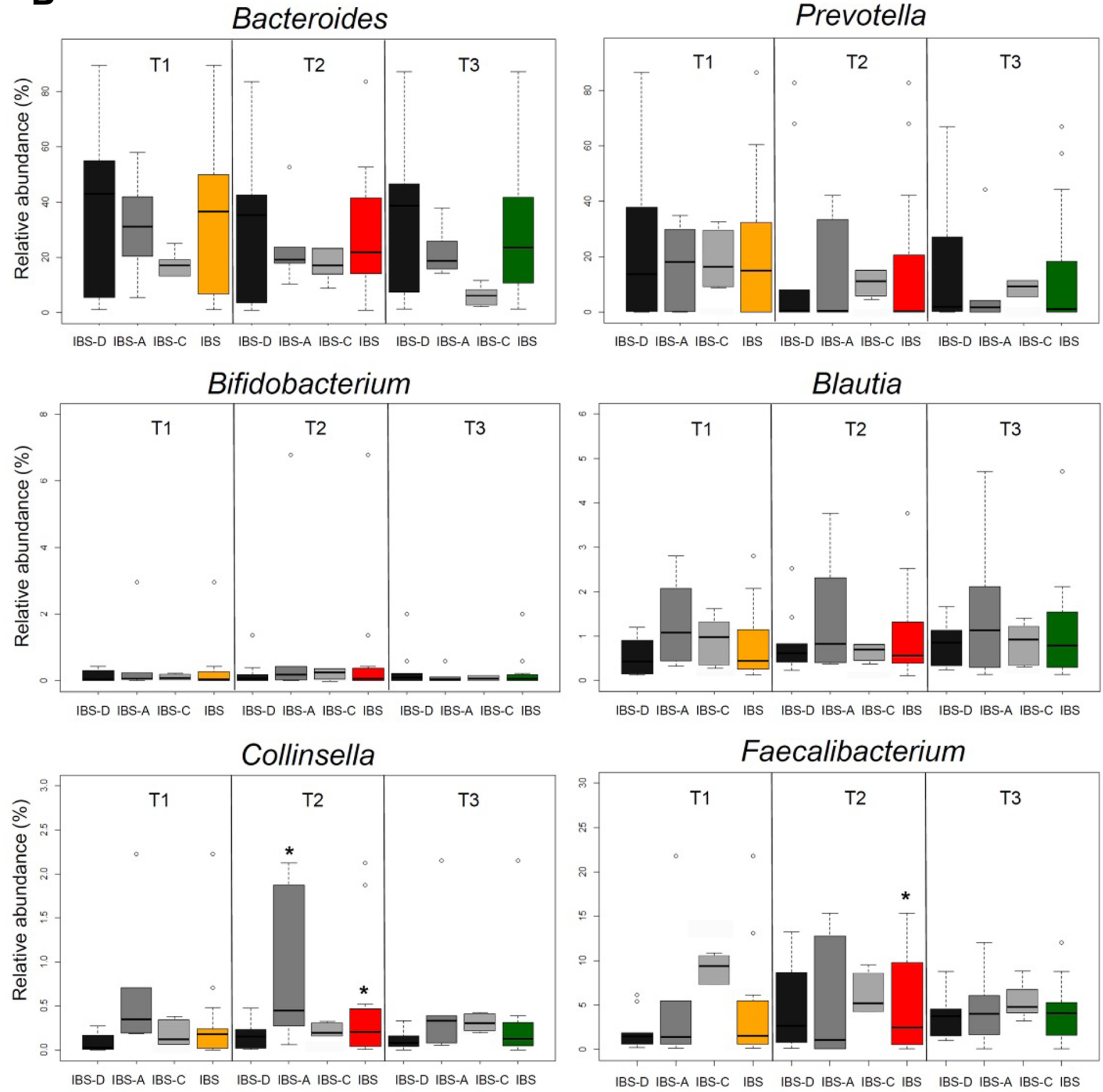

Faecalibacterium

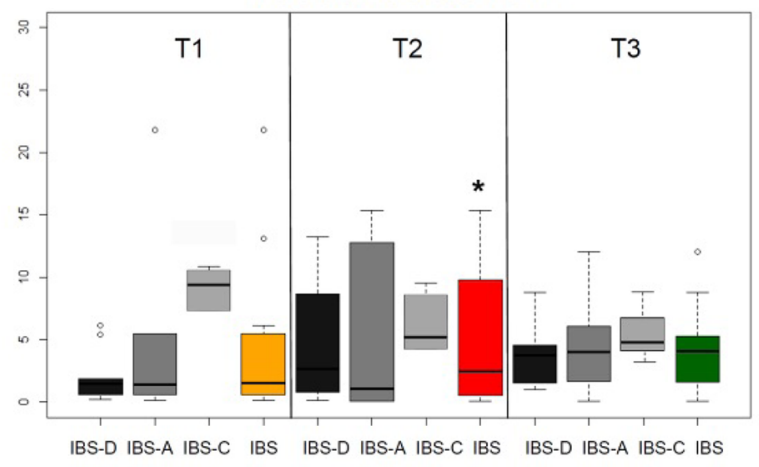

Fig. 5 (See legend on next page.) 
(See figure on previous page.)

Fig. 5 Impact of geraniol-based intervention on the intestinal microbiota structure in IBS patients. a Principal component analysis of Euclidean distances between the genus-level intestinal microbial profiles of IBS patients at the baseline (orange), after four-week intervention (red), and at follow-up after a further four weeks (olive green). Ellipses include the $99 \%$ confidence area based on the standard error of the weighted average of sample coordinates. A trend towards decreasing PC2 values after the intervention was observed (Wilcoxon test, $P=0.14$ ). $\mathbf{b}$ Box plots showing the distribution of the relative abundance values of IBS discriminant taxa over time (T1, baseline; T2, after four-week intervention; T3, at follow-up). Data are shown for the whole IBS cohort and for IBS subtypes. The increase in the relative abundance of Collinsella and Faecalibacterium at T2 compared to the baseline (T1) was statistically significant ${ }^{*}$, Wilcoxon test, $P=0.04$ )

evidenced following oral administration in rodents of doses ranging from $50 \mathrm{mg} \mathrm{kg}^{(-1)}$ die to more than 200 $\mathrm{mg} \mathrm{kg}{ }^{(-1)}$ die $[20,23,33]$. Nevertheless, to the best of our knowledge, this pilot study was the first to administer geraniol orally to humans. The potential therapeutic use of geraniol to target gut dysbiosis and inflammation appears promising, but in its free form, $\mathrm{Ge}-\mathrm{OH}$ is easily absorbed in the small intestine and does not reach the colon except in very small quantities [26]. Therefore, $\mathrm{Ge}-\mathrm{OH}$ must be associated with vehicles capable of maximizing its delivery into the colon.

This study evaluated the capacity of $\mathrm{Ge}-\mathrm{OH}$ delivered to the large intestine in soy lecithin micelles to reduce symptomatology in IBS patients by modulating the gut microbiota known to be strongly altered in this pathology [13]. In particular, Ge-OH treatment resulted in increased relative abundances of Collinsella and Faecalibacterium. Interestingly, a reduction in the amount of Collinsella aerofaciens was previously observed in the fecal microbiota of IBS patients compared with healthy controls, and the decreased abundance of Collinsella has been associated with the severity of IBS symptoms [34]. On the other hand, the well-known short-chain fatty acid producer Faecalibacterium is among the most representative species found to be decreased in IBS and many other intestinal and metabolic diseases [35]. Since no patient before and during the trial used a particular diet we can exclude a "diet effect" on symptomatology improvement and microbiota modifications.

$\mathrm{Ge}-\mathrm{OH}$ administration also led to increased ecosystem biodiversity in IBS patients. Even if this modulation was not statistically significant, probably due to the small number of patients enrolled and large inter-individual differences, the increased diversity was still detectable at T3, suggesting a lasting $\mathrm{Ge}-\mathrm{OH}$ effect on microbiota structure. This effect is not in contrast with $\mathrm{Ge}-\mathrm{OH}$ antimicrobial activity, since it is well established that its IC50 values vary a lot between different bacterial genera and species [20]. Indeed, genera such as Bacteroides and Prevotella, found to be enriched in IBS, decreased their relative abundance following $\mathrm{Ge}-\mathrm{OH}$ treatment, even if statistical significance was not achieved. It is noteworthy that a similar pilot study in 19 IBS patients reported that the IBS-associated imbalance of the intestinal microbiota was not reverted by VLS\#3 probiotic supplementation [30].
The pathogenesis of IBS has also been linked to a low grade of gut and systemic inflammation [36]. Furthermore, levels of chemotactic chemokines, such as monocyte chemoattractant protein-1 (MCP-1/CCL2), macrophage

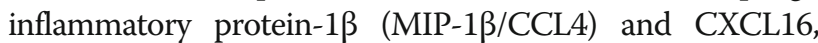
were found to be higher in the sera and stools of idiopathic IBS patients [8]. Overall, it is reasonable that a sort of self-sustaining inflammatory loop between the gut microbiota and low-grade gastrointestinal inflammation exists in IBS patients. Our results show that $\mathrm{Ge}-\mathrm{OH}$ treatment is capable of significantly reducing serum MIP-1 $\beta$ at T2. MCP-1, IL-6 and IL-17A were also modulated by Ge-OH at T2, but their decrease was only close to reaching statistical relevance. These antinflammatory effects may be driven by the more protective profile of the intestinal community induced by $\mathrm{Ge}-\mathrm{OH}$ modulation of gut bacteria. Data obtained in this trial are consistent with our previous experiments in mice, where $\mathrm{Ge}-\mathrm{OH}$ oral administration using soy lecithin micelles resulted in a consistent decrease in pro-inflammatory circulating cytokines associated with a positive microbiota modulation in dextran sulfate sodium (DSS)-induced colitis [24]. This effect was particularly strong when $\mathrm{Ge}-\mathrm{OH}$ was administered directly into the colon by enema. In rat, soy lecithin micelles deliver only $50 \%$ of orally administered $\mathrm{Ge}-\mathrm{OH}$ to the large bowel since $50 \%$ is adsorbed in the small intestine [26]. It is therefore presumable that in this formulation only an estimated 4 $\mathrm{mg} \mathrm{kg} \mathrm{g}^{(-1)}$ die of geraniol effectively reached the colon of our IBS patients.

We are conscious that, since the VAS-IBS score is based on patients' self-reported symptoms, it cannot quantify the placebo effect that in this particular pathology is known to range between 37 to $47 \%$ for pharmacological treatments and complementary medicine [37]. Despite this, the fecal microbiota analysis showed that $\mathrm{Ge}-\mathrm{OH}$ is able to partially revert dysbiosis in this cohort of patients. We are aware that our study has important limitations, namely the absence of a double-blind placebo arm. Moreover, the number of patients enrolled is small. On the other hand, we carefully selected patients, and the results in terms of symptomatology, reduced dysbiosis and decreased pro-inflammatory chemokines are certainly promising, especially for IBS-D subtypes, in which $\mathrm{Ge}-\mathrm{OH}$ effect on symptomatology seems to be maintained four weeks after taking the last dose of this monoterpene. 


\section{Conclusions}

The data obtained from this study are promising and statistically significant in terms of microbiota modulation, decrease of circulating MIP-1 $\beta$ and reduced VAS-IBS score. A placebo-controlled study on a larger population is now needed to confirm the effectiveness of $\mathrm{Ge}-\mathrm{OH}$ in improving the symptomatology of IBS patients. Nevertheless, food supplement formulations enriched in $\mathrm{Ge}-\mathrm{OH}$ and capable of delivering it to the large bowel could be used to counteract or prevent dysbiosis.

\section{Abbreviations}

DNEL: Derived No Effect Level; FODMAPs: Fermentable Oligo-, Di- and Mono- saccharides And Polyols; Ge-OH: Geraniol; GRAS: Generally Recognized As Safe; IBS: Irritable Bowel Syndrome; VAS-IBS: Visual Analogue Scale for Irritable Bowel Syndrome

\section{Acknowledgements}

The authors thank Dr. A. Sardo for his technical and moral support.

\section{Funding}

This study was supported by the University of Bologna (RFO 2015), Xeda international (1397 Route nationale 7, Zac la Crau, 13670 Saint Andiol, France).

\section{Availability of data and materials \\ Sequencing reads have been deposited in SOURCEFORGE (https:// sourceforge.net/projects/geraniol-in-ibs/). All other data were collected in pseudo-anonymous form, no explicit consent was given by patients to provide raw data to third parties not involved in the clinical trial.}

\section{Authors' contributions}

$\mathrm{FR}$, CR, and MasC contributed to patient enrollment, study design and interpretation of clinical data. MS, EG and EC contributed to patients enrollment, VAS-IBS questionnaire administration, data monitoring, data management and statistical analyses of VAS-IBS data. AC and LDF contributed to sample collection, DNA extraction from stool samples, Cytokines and chemokines Luminex determination, data interpretation and statistical analysis. MarC and ST performed microbiota analyses and interpretation of data. MCV and ES contributed to the conception and design of the study, drafted and revised the paper. All authors have read the manuscript and gave final approval of the version to be published.

\section{Ethics approval and consent to participate}

Patients were informed of the full nature and purpose of the study, and provided written informed consent before entering the trial. The study was conducted in conformity with the principles of Declaration of Helsinki and Good Clinical Practice. The study was approved by the Ethics Committee of the AOU Policlinico S. Orsola-Malpighi; CE code 100/2013/U/Sper and by the Ethics Committee of the ASST Spedali Civili di Brescia; CE code NP2047.

\section{Consent for publication}

All patients gave explicit written consent for data publication in aggregated form.

\section{Competing interests}

The authors declare that the research was conducted in the absence of any commercial or financial relationships that could be construed as a potential conflict of interest.

\section{Publisher's Note}

Springer Nature remains neutral with regard to jurisdictional claims in published maps and institutional affiliations.

\section{Author details}

'Department of Medical and Surgical Sciences, University of Bologna, Via Massarenti 9, 40138 Bologna, Italy. ${ }^{2}$ Department of Clinical and Experimental Sciences, University of Brescia, Spedali Civili 1, 25121 Brescia, Italy. ${ }^{3}$ Department of Biological, Geological and Environmental Sciences, Biology Unit, University of Bologna, Via Selmi 3, 40126 Bologna, Italy. ${ }^{4}$ Department of
Pharmacy and Biotechnology, University of Bologna, Via Belmeloro 6, 40126 Bologna, Italy.

Received: 15 June 2018 Accepted: 6 December 2018

Published online: 19 December 2018

\section{References}

1. Canavan C, West J, Card T. The epidemiology of irritable bowel syndrome. Clin Epidemiol. 2014;6:71-80.

2. Buono $J$, Carson RT, Flores NM. Health-related quality of life, work productivity, and indirect costs among patients with irritable bowel syndrome with diarrhea. Health Qual Life Outcomes. 2017;15:35.

3. Holten $\mathrm{KB}$, Wetherington A, Bankston L. Diagnosing the patient with abdominal pain and altered bowel habits: is it irritable bowel syndrome? Am Fam Physician. 2003;67:2157-62.

4. Drossman DA, Corazziari E, Talley NJ, Thompson WG, Whitehead WE. Rome II: a multinational consensus document on functional gastrointestinal disorders. Gut. 1999:45:SII II1-||81.

5. Bai T, Xia J, Jiang Y, Cao H, Zhao Y, Zhang L, Wang H, Song J, Hou X. Comparison of the Rome IV and Rome III criteria for IBS diagnosis: a crosssectional survey. J Gastroenterol Hepatol. 2017;32:1018-25.

6. Saha L. Irritable bowel syndrome: pathogenesis, diagnosis, treatment, and evidence-based medicine. World J Gastroenterol. 2014;20:6759-73.

7. Bashashati M, Rezaei N, Andrews CN, Chen CQ, Daryani NE, Sharkey KA, Storr MA. Cytokines and irritable bowel syndrome: where do we stand? Cytokine. 2012;57:201-9.

8. Darkoh C, Comer L, Zewdie G, Harold S, Snyder N, Dupont HL. Chemotactic chemokines are important in the pathogenesis of irritable bowel syndrome. PLoS One. 2014;9:e93144.

9. Collins SM. A role for the gut microbiota in IBS. Nat Rev Gastroenterol Hepatol. 2014;11:497-505.

10. Jeffery IB, O'Toole PW, Öhman L, Claesson MJ, Deane J, Quigley EM, Simrén M. An irritable bowel syndrome subtype defined by species-specific alterations in faecal microbiota. Gut. 2012;61:997-1006.

11. Rajilić-Stojanović M, Biagi E, Heilig HG, Kajander K, Kekkonen RA, Tims S, de Vos WM. Global and deep molecular analysis of microbiota signatures in fecal samples from patients with irritable bowel syndrome. Gastroenterology. 2011;141:1792-801

12. Bhattarai Y, Muniz Pedrogo DA, Kashyap PC. Irritable bowel syndrome: a gut microbiota-related disorder? Am J Physiol Gastrointest Liver Physiol. 2017; 312:G52-62.

13. Zhang Y, Li L, Guo C, Mu D, Feng B, Zuo X, Li Y. Effects of probiotic type, dose and treatment duration on irritable bowel syndrome diagnosed by Rome III criteria: a meta-analysis. BMC Gastroenterol. 2016;16:62.

14. Staudacher HM, Whelan K. Altered gastrointestinal microbiota in irritable bowel syndrome and its modification by diet: probiotics, prebiotics and the low FODMAP diet. Proc Nutr Soc. 2016;75:306-18.

15. Currò D, laniro G, Pecere S, Bibbò S, Cammarota G. Probiotics, fibre and herbal medicinal products for functional and inflammatory bowel disorders. Br J Pharmacol. 2016:174:1426-49.

16. Ponziani FR, Pecere S, Lopetuso L, Scaldaferri F, Cammarota G, Gasbarrini A. Rifaximin for the treatment of irritable bowel syndrome - a drug safety evaluation. Expert Opin Drug Saf. 2016;15:983-91.

17. Oviedo-Rondón EO, Hume ME, Hernández C, Clemente-Hernández S. Intestinal microbial ecology of broilers vaccinated and challenged with mixed Eimeria species, and supplemented with essential oil blends. Poult Sci. 2006;85:854-60.

18. Thompson A, Meah D, Ahmed N, Conniff-Jenkins R, Chileshe E, Phillips CO, Claypole TC, Forman DW, Row PE. Comparison of the antibacterial activity of essential oils and extracts of medicinal and culinary herbs to investigate potential new treatments for irritable bowel syndrome. BMC Complement Altern Med. 2013;13:338.

19. Khan AQ, Khan R, Qamar W, Lateef A, Rehman MU, Tahir M, Ali F, Hamiza OO, Hasan SK, Sultana S. Geraniol attenuates 12-Otetradecanoylphorbol-13-acetate (TPA)-induced oxidative stress and inflammation in mouse skin: possible role of p38 MAP kinase and NFkappaB. Exp Mol Pathol. 2013:94:419-29.

20. Thapa D, Losa R, Zweifel B, Wallace RJ. Sensitivity of pathogenic and commensal bacteria from the human colon to essential oils. Microbiology. 2012;158:2870-7. 
21. Di Pasqua R, Hoskins N, Betts G, Mauriello G. Changes in membrane fatty acids composition of microbial cells induced by addition of thymol, carvacrol, limonene, cinnamaldehyde, and eugenol in the growing media. J Agric Food Chem. 2006;54:2745-9.

22. Turina AV, Nolan MV, Zygadlo JA, Perillo MA. Natural terpenes: self-assembly and membrane partitioning. Biophys Chem. 2006;122:101-13.

23. Solorzano-Santos F, Miranda-Novales MG. Essential oils from aromatic herbs as antimicrobial agents. Curr Opin Biotechnol. 2012;23:136-41.

24. De Fazio L, Spisni E, Cavazza E, Strillacci A, Candela M, Centanni M, Ricci C, Rizzello F, Campieri M, Valerii MC. Dietary geraniol by Oral or Enema administration strongly reduces Dysbiosis and systemic inflammation in dextran sulfate sodium-treated mice. Front Pharmacol. 2016;7:38.

25. Medicherla K, Sahu BD, Kuncha M, Kumar JM, Sudhakar G, Sistla R. Oral administration of geraniol ameliorates acute experimental murine colitis by inhibiting pro-inflammatory cytokines and NF-KB signaling. Food Funct. 2015;6:2984-95.

26. Pavan B, Dalpiaz A, Marani L, Beggiato S, Ferraro L, Canistro D, Paolini M, Vivarelli F, Valerii MC, Comparone A, De Fazio L, Spisni E. Geraniol pharmacokinetics, bioavailability and its multiple effects on the liver antioxidant and xenobiotic-metabolizing enzymes. Front Pharmacol. 2018;9:18.

27. Turroni S, Fiori J, Rampelli S, Schnorr SL, Consolandi C, Barone M, Biagi E, Fanelli F, Mezzullo M, Crittenden AN, Henry AG, Brigidi P, Candela M. Fecal metabolome of the Hadza hunter-gatherers: a host-microbiome integrative view. Sci Rep. 2016;6:32826.

28. Swanson KS, Dowd SE, Suchodolski JS, Middelbos IS, Vester BM, Barry KA, Nelson KE, Torralba M, Henrissat B, Coutinho PM, Cann IK, White BA, Fahey GC $J$ J. Phylogenetic and gene-centric metagenomics of the canine intestinal microbiome reveals similarities with humans and mice. ISME J. 2011;5:639-49.

29. Edgar RC. Search and clustering orders of magnitude faster than BLAST. Bioinformatics. 2010;26:2460-1.

30. Maccaferri S, Candela M, Turroni S, Centanni M, Severgnini M, Consolandi C, Cavina P, Brigidi P. IBS-associated phylogenetic unbalances of the intestinal microbiota are not reverted by probiotic supplementation. Gut Microbes. 2012;:406-13

31. Sonnenburg ED, Sonnenburg JL. Starving our microbial self: the deleterious consequences of a diet deficient in microbiota-accessible carbohydrates. Cell Metab. 2014;20:779-86.

32. Lapczynski A, Bhatia SP, Foxenberg RJ, Letizia CS, Api AM. Fragrance material review on geraniol. Food Chem Toxicol. 2008:46:S160-70

33. Rekha KR, Selvakumar GP, Sethupathy S, Santha K, Sivakamasundari RI. Geraniol ameliorates the motor behavior and neurotrophic factors inadequacy in MPTP-induced mice model of Parkinson's disease. J Mol Neurosci. 2013;51:851-62

34. Malinen $E$, Krogius-Kurikka L, Lyra A, Nikkilä J, Jääskeläinen $A$, Rinttilä T, Vilpponen-Salmela T, von Wright AJ, Palva A. Association of symptoms with gastrointestinal microbiota in irritable bowel syndrome. World J Gastroenterol. 2010;16:4532-40.

35. Liu HN, Wu H, Chen YZ, Chen YJ, Shen XZ, Liu TT. Altered molecular signature of intestinal microbiota in irritable bowel syndrome patients compared with healthy controls: a systematic review and meta-analysis. Dig Liver Dis. 2017:49:331-7.

36. Dinan TG, Clarke G, Quigley EM, Scott LV, Shanahan F, Cryan J, Cooney J, Keeling PW. Enhanced cholinergic-mediated increase in the proinflammatory cytokine IL-6 in irritable bowel syndrome: role of muscarinic receptors. Am J Gastroenterol. 2008;103:2570-6.

37. Flik CE, Bakker L, Laan W, van Rood YR, Smout AJ, de Wit NJ. Systematic review: the placebo effect of psychological interventions in the treatment of irritable bowel syndrome. World J Gastroenterol. 2017;23:2223-33.

\section{Ready to submit your research? Choose BMC and benefit from}

- fast, convenient online submission

- thorough peer review by experienced researchers in your field

- rapid publication on acceptance

- support for research data, including large and complex data types

- gold Open Access which fosters wider collaboration and increased citations

- maximum visibility for your research: over $100 \mathrm{M}$ website views per year

At BMC, research is always in progress.

Learn more biomedcentral.com/submissions 